\title{
Neutral $B$-meson and $D$-meson mixing matrix elements from $2+1$ flavor lattice $Q C D$
}

\author{
C. Bernard ${ }^{a}$, C.M. Bouchard ${ }^{b}$, C.C. Chang ${ }^{c}$, A.X. El-Khadrac ${ }^{c}$, E.D. Freeland ${ }^{d}$, \\ E. Gámiz ${ }^{e}$, A.S. Kronfeld ${ }^{f, g}$, J. Laiho ${ }^{h}$, P.B. Mackenzie ${ }^{f}$, E.T. Neil ${ }^{i, j}$, J.N. Simone ${ }^{*, f, \alpha}$, \\ and R.S. Van de Water ${ }^{f}$ \\ ${ }^{a}$ Department of Physics, Washington University, St. Louis, MO 63130, USA \\ ${ }^{b}$ Physics Department, The College of William and Mary, Williamsburg, VA 23187, USA \\ ${ }^{c}$ Department of Physics, University of Illinois, Urbana, IL 61801, USA \\ ${ }^{d}$ Liberal Arts Department, School of the Art Institute of Chicago, Chicago, IL 60603, USA \\ ${ }^{e}$ CAFPE and Depto. de Física Teórica y del Cosmos, Universidad de Granada, Granada, España \\ ${ }^{f}$ Fermi National Accelerator Laboratory, Batavia, IL 60510, USA \\ ${ }^{g}$ Institute for Advanced Study, Technische Universität München, Garching, Germany \\ ${ }^{h}$ Department of Physics, Syracuse University, Syracuse, NY 13244, USA \\ ${ }^{i}$ Department of Physics, University of Colorado, Boulder, CO 80309, USA \\ ${ }^{j}$ RIKEN-BNL Research Center, Brookhaven National Laboratory, Upton, NY 11973, USA

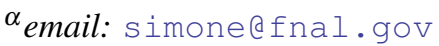

\section{Fermilab Lattice and MILC Collaborations}

\begin{abstract}
We compute the hadronic matrix elements of the complete set of local four-quark operators relevant for neutral $B$-meson mixing in the Standard Model and beyond as well as for the the shortdistance contributions to $D^{0}$ mixing. In addition we calculate the leptonic decay constants for the $B$ - and $D$-meson systems on the same sets of gauge-field ensembles. Both mixing matrix elements and decay constants are computed with asqtad-improved light quarks and clover heavy quarks in the Fermilab interpretation. We use the MILC three-flavor asqtad ensembles at four lattice spacings between appoximately $0.11 \mathrm{fm}$ and $0.043 \mathrm{fm}$. We present nearly final values for the mixing matrix elements, and find for the $S U(3)$ breaking ratio $\xi=1.203(17)(6)_{\text {sea }_{c}}$. The second error accounts for the omission of charm quarks in the sea while the first error includes all other sources of uncertainty. Our result for $\xi$ when combined with the experimentally measured frequencies gives $\left|V_{t d}\right| /\left|V_{t s}\right|=0.2047(4)_{\exp }(29)_{\text {th }}(10)_{\text {sea }_{c}}$. Finally, we report on our progress towards finalizing our decay constant and bag parameter analyses.
\end{abstract}

The 33rd International Symposium on Lattice Field Theory

14 - 18 July 2015

Kobe International Conference Center, Kobe, Japan*

${ }^{*}$ Speaker. 


\section{Introduction}

The neutral $B$-meson mixing rates are sensitive probes of physics at energy scales beyond the direct reach of current particle accelerators. This sensitivity arises because at leading order in the Standard Model these decays occur though "box diagrams" involving virtual $W$ bosons and virtual top quarks. Physics beyond the Standard Model can potentially contribute significantly to neutral meson mixing, for example via the exchange of new heavy particles.

In the Standard Model, the $B_{q}^{0}$-mixing rate $(q=d, s)$ becomes

$$
\Delta M_{q}=\text { (known factor) }\left|V_{t q}^{*} V_{t b}\right|^{2}\left\langle B_{q}^{0}\left|\mathscr{O}_{1}^{q}\right| \bar{B}_{q}^{0}\right\rangle,
$$

where "known factor" includes the short-distance effects while the long-distance effects arise from the hadronic matrix element of a single, local, four-quark effective operator $\mathscr{O}_{1}^{q}$. The CKM matrix elements $\left|V_{t d}\right|$ and $\left|V_{t s}\right|$ are then determined from experimental measurements of the mixing frequencies in combination with the hadronic matrix elements from lattice QCD. Moreover, the ratio of $B$-meson mixing frequencies determines $\left|V_{t d}\right| /\left|V_{t s}\right|$ which constrains the apex of the CKM unitarity triangle used to test the consistency of experiment and the Standard Model. A basis of five local effective operators:

$$
\begin{array}{lll}
1:\left(\bar{b}^{\alpha} \gamma_{\mu} L q^{\alpha}\right)\left(\bar{b}^{\beta} \gamma_{\mu} L q^{\beta}\right) & 2:\left(\bar{b}^{\alpha} L q^{\alpha}\right)\left(\bar{b}^{\beta} L q^{\beta}\right) & 3:\left(\bar{b}^{\alpha} L q^{\beta}\right)\left(\bar{b}^{\beta} L q^{\alpha}\right) \\
4:\left(\bar{b}^{\alpha} L q^{\alpha}\right)\left(\bar{b}^{\beta} R q^{\beta}\right) & 5:\left(\bar{b}^{\alpha} L q^{\beta}\right)\left(\bar{b}^{\beta} R q^{\alpha}\right)
\end{array}
$$

is sufficient to describe mixing at leading order in the SM and any extension thereof $[1,2]$.

We report on our ongoing lattice QCD calculation of the $B_{q}^{0}$-mixing matrix elements as well as the analogous set of matrix elements that describe the short-distance contributions to $D^{0}-\bar{D}^{0}$ mixing. We update the status of this computation last reported in Refs. [3, 4]. Progress since our last report includes finalizing the analysis of the statistical and systematic effects. We present nearly final results for the matrix elements and the ratio $\xi$. We report progress towards bag parameters computed from ratios of the mixing matrix elements and the leptonic decay constants including both statistical and systematic correlations. For that purpose the same approach to error analysis and the same bootstrap resampling is used in both analyses.

\section{Mixing matrix elements}

The mixing matrix elements are extracted from an analysis of three-point and two-point functions computed on each of the MILC $2+1$ flavor asqtad gauge ensembles listed in Table 1. On each ensemble, the $B_{q}$ and $D_{q}$ correlators are computed for seven or eight values of the light valencequark mass, $m_{q}$, spanning the range: $\min \left(m_{l}, 0.1 m_{h}\right) \lesssim m_{q} \lesssim m_{h}$, where $m_{l}$ and $m_{h}$ are the sea quark masses. More details about methods and procedures used to extract the mixing matrix elements from the correlation functions are in Ref. [29].

We adopt a mostly nonperturbative renormalization ( $\mathrm{mNPR}$ ) procedure to match lattice results to the continuum [30]. The nonperturbative factors $Z_{V_{Q Q}^{4}}$ (heavy) and $Z_{V_{q q}^{4}}$ (light) match the flavorconserving vector currents. In terms of these factors, the matching of the four-fermion mixing operators is:

$$
\left\langle\mathscr{O}_{i}\right\rangle=Z_{V_{Q Q}^{4}} Z_{V_{q q}^{4}}\left[\delta_{i j}+\alpha_{s} \rho_{i j}^{[1]}\right]\left\langle\mathscr{O}_{j}\right\rangle^{\text {lat }}
$$




\begin{tabular}{lllllllrcc}
\hline id & $\mathbf{a}[\mathbf{f m}]$ & $\mathbf{b e t a}$ & $\mathbf{m}_{\mathbf{l}} / \mathbf{m}_{\mathbf{h}}$ & $\mathbf{a m}_{\mathbf{h}}$ & $\mathbf{m}_{\mathbf{h}} / \mathbf{m}_{\mathbf{s}}$ & $\mathbf{r}_{\mathbf{1}} / \mathbf{a}$ & $\mathbf{N}_{\text {config }}$ & $\mathbf{N}_{\text {tsrc }}$ & $\mathbf{D O I}$ \\
\hline en24 & 0.043 & 7.81 & 0.2 & 0.014 & 1.079 & 7.208 & 801 & 4 & {$[5]$} \\
en18 & 0.059 & 7.46 & 0.1 & 0.018 & 1.019 & 5.307 & 827 & 4 & {$[6,7]$} \\
en19 & 0.058 & 7.465 & 0.139 & 0.018 & 1.024 & 5.330 & 801 & 4 & {$[8]$} \\
en20 & 0.058 & 7.47 & 0.2 & 0.018 & 1.028 & 5.353 & 673 & 8 & {$[9,10]$} \\
en23 & 0.058 & 7.48 & 0.4 & 0.018 & 1.037 & 5.399 & 593 & 4 & {$[11,12]$} \\
en12 & 0.083 & 7.075 & 0.05 & 0.031 & 1.255 & 3.738 & 791 & 4 & {$[13]$} \\
en13 & 0.083 & 7.08 & 0.1 & 0.031 & 1.256 & 3.755 & 1015 & 4 & {$[14,15]$} \\
en14 & 0.083 & 7.085 & 0.15 & 0.031 & 1.262 & 3.772 & 984 & 4 & {$[16]$} \\
en15 & 0.082 & 7.09 & 0.2 & 0.031 & 1.267 & 3.789 & 1931 & 4 & {$[17-19]$} \\
en17 & 0.081 & 7.11 & 0.4 & 0.031 & 1.290 & 3.858 & 1996 & 4 & {$[20]$} \\
en04 & 0.11 & 6.76 & 0.1 & 0.05 & 1.489 & 2.739 & 2099 & 4 & {$[21]$} \\
en05 & 0.11 & 6.76 & 0.14 & 0.05 & 1.489 & 2.739 & 2110 & 4 & {$[22,23]$} \\
en06 & 0.11 & 6.76 & 0.2 & 0.05 & 1.489 & 2.739 & 2259 & 4 & {$[24,25]$} \\
en09 & 0.11 & 6.79 & 0.4 & 0.05 & 1.534 & 2.821 & 2052 & 4 & {$[26,27]$} \\
\hline
\end{tabular}

Table 1: MILC $2+1$ flavor asqtad ensembles at four lattice spacings used to compute mixing matrix elements and the heavy-light decay constants [5-28]. Mass $m_{h}$ is the simulated strange quark mass and $m_{l}$ is the mass of the two light flavors. Ensemble "en12" is near the physical mass point: $m_{l} / m_{h}=1 / 20$.

where the one-loop corrections, $\rho_{i j}^{[1]}$, are known in perturbation theory. We allow for higher order $\alpha_{s}^{2} \rho_{i j}^{[2]}$ and $\alpha_{s}^{3} \rho_{i j}^{[3]}$ terms in the fits described below, where the unknown $\rho_{i j}^{[n]}$ are constrained via priors.

We perform a combined continuum and chiral extrapolation of lattice $B_{q}$ (or $D_{q}$ ) results to obtain the matrix elements at physical values of the quark masses. All five matrix elements are included in the combined fit with the fit function given schematically as

$$
\begin{aligned}
\left\langle\mathscr{O}_{i}\right\rangle & =\beta_{i}\left[1+\chi \operatorname{logs}{ }_{i}+\text { "wrong spin" logs }_{i}\right]+\beta_{j}^{\prime} \text { "wrong spin" logs }_{i j}+\text { analytic }_{i} \\
& + \text { HQ mass tuning }_{i}+\text { HQ discretization }_{i}+\text { LQ disc. }_{i}+\beta_{j} \text { H.O. matching }_{i j}
\end{aligned}
$$

where the subsets of operators with indices $i, j \in 1,2,3: j \neq i$ and $i, j \in 4,5: j \neq i$ mix. Coefficients $\beta_{i}$ and $\beta_{j}^{\prime}$ are low energy constants (LECs) of chiral perturbation theory, additional LECs and other fit parameters appear within the terms shown. The fit function includes the NLO chiral logarithm and analytic terms of "rooted, heavy-meson staggered chiral perturbation theory" [31]. The "wrong spin" logarithmic terms are artifacts from staggered fermions; fortunately, they do not lead to any new LECs. Analytic terms also include NNLO as well as $\mathrm{N}^{3} \mathrm{LO}$ terms which are used as a check of systematic effects from truncating the chiral expansion. Leading $O\left(\alpha_{s} a \Lambda\right)$ and $O\left(a^{2} \Lambda^{2}\right)$ heavy quark discretization effects are modeled by terms in the fit function. The heavy-quark (HQ) mass tuning term adjusts for any mistuning of the charm or bottom quark mass used to calculate the matrix elements. The higher-order matching includes $O\left(\alpha_{s}^{2}\right)$ terms as well as $O\left(\alpha_{s}^{3}\right)$ terms used to check systematics from truncating the matching.

Parameters of the fits are constrained by Gaussian priors. We minimize the fully-covariant weighted sum of squared residuals - the "Chi-square" error function, augmented by the prior constraints, to find the optimal fit [33]. A bootstrap resampling process propagates errors and preserves the correlations in fits to correlator data. In the chiral fits, errors are propagated and correlations 


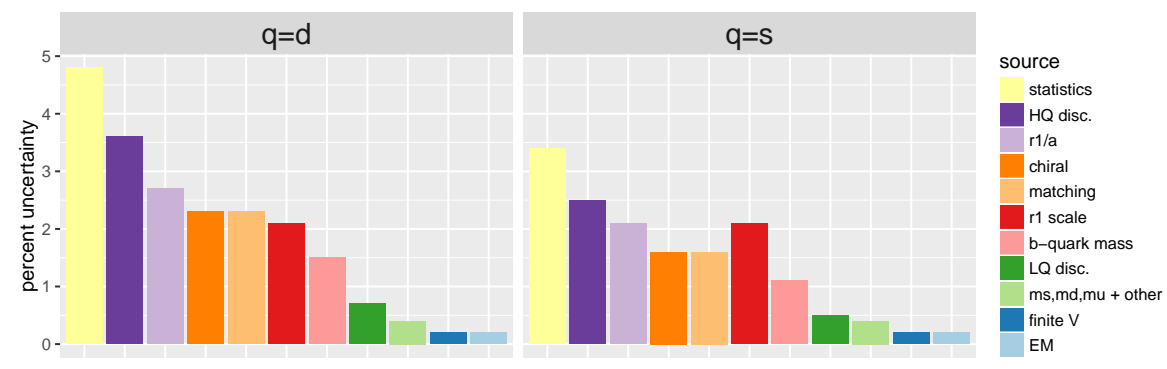

Figure 1: Estimated uncertainty breakdown of the fit error and errors from $r_{1}, \mathrm{FV}$ and EM for the $B_{q}^{0}$ mixing matrix elements $\left\langle\mathscr{O}_{1}^{q}\right\rangle$. The errors are summed in quadrature, following the procedure in Ref. [32] .

preserved as described in Ref. [32]. We quote values and errors for the matrix elements from a fit that we label the "base" fit. This fit includes the NNLO analytic terms, matching through $O\left(\alpha_{s}^{2}\right)$, and our nominal choices for the prior widths. The total fit error includes both statistics and uncertainties from the systematic effects modeled in the fit function. The relative importance of the sources of statistical and systematic error, with correlations, are found from the fitted model. Figure 1 depicts the relative importance of the sources of error for the $B_{q}^{0}$ mixing matrix elements $\left\langle\mathscr{O}_{1}^{q}\right\rangle$. The leading errors suggest a program for reducing errors in future calculations: better statistics, runs on finer lattice spacings, a more precise method for setting of the distance scale using, e.g., Wilson Flow techniques, and including ensembles at or near the physical quark masses.

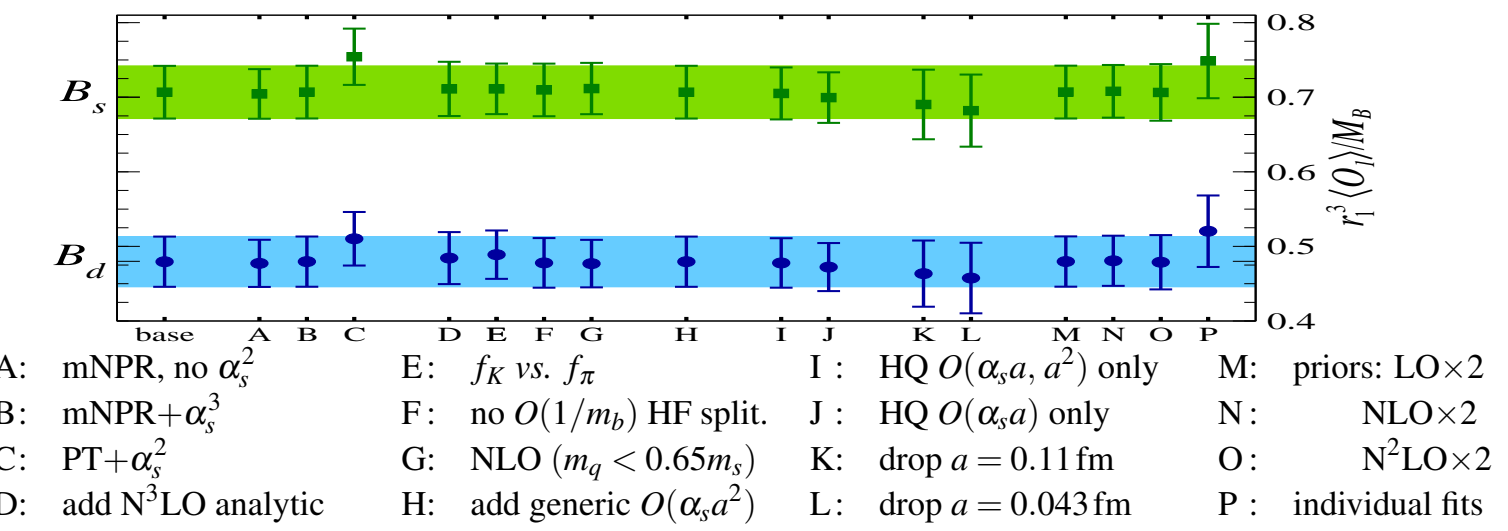

Figure 2: Stability of the $\left\langle\mathscr{O}_{1}^{q}\right\rangle$ from the base fit with respect to the listed variations in the fitting procedure. The green and blue bands depict the total error from the base fit, fit variations lie within these bands.

We perform alternate fits where we vary the fit function, input parameters, prior widths and data to test if the "base" fit fully account for all of the uncertainties. Figure 2 shows little variation in results from alternate fits for $\left\langle\mathscr{O}_{1}^{q}\right\rangle$ for $B_{q}^{0}$ with respect to the base fit, demonstrating stability. The full set operators for $B_{q}^{0}$ as well as $D^{0}$ show similar stability. Tested variations in the matching procedure include: A) omitting $O\left(\alpha_{s}^{2}\right)$ terms, B) allowing for $O\left(\alpha_{s}^{3}\right)$ contributions, and C) replacing mNPR matching by purely perturbative matching. Variations in the chiral expansion include: D) the addition of $\mathrm{N}^{3} \mathrm{LO}$ analytic terms, E) changing the pseudoscalar decay constant value in the chiral expansion, F) omitting the $B^{*}-B$ hyperfine splitting (an $O\left(1 / m_{B}\right)$ effect) in the chiral logarithms, and G) truncation of the chiral expansion to NLO order while restricting $m_{q}<0.65 m_{s}$. 


\begin{tabular}{|c|c|c|c|}
\hline matrix & $B^{0}-\bar{B}^{0}$ & $B_{s}^{0}-\bar{B}_{s}^{0}$ & $D^{0}-\bar{D}^{0}$ \\
\hline element & BBGLN & BBGLN & BBGLN BMU \\
\hline$\left\langle\mathscr{O}_{1}\right\rangle$ & $0.645(50)(13)$ & $0.965(56)(19)$ & $0.0715(62)(14)$ \\
\hline$\left\langle\mathscr{O}_{2}\right\rangle$ & $-0.565(46)(11)-0.537(44)(11)$ & $-0.860(51)(17)-0.811(48)(16)$ & $-0.148(8)(3)-0.137(7)(3)$ \\
\hline$\left\langle\mathscr{O}_{3}\right\rangle$ & $0.151(22)(3) \quad 0.152(23)(3)$ & $0.221(23)(4) \quad 0.223(23)(4)$ & $0.056(4)(1) \quad 0.054(4)(1)$ \\
\hline$\left\langle\mathscr{O}_{4}\right\rangle$ & $0.970(67)(19)$ & $1.363(74)(27)$ & $0.280(14)(6)$ \\
\hline$\left\langle\mathscr{O}_{5}\right\rangle$ & $0.525(45)(10)$ & $0.737(48)(15)$ & $0.104(9)(2)$ \\
\hline & renorm. sc & le: $\mu=m_{b}$ & $\mu=3 \mathrm{GeV}$ \\
\hline
\end{tabular}

Table 2: Results for $\overline{\mathrm{MS}}-\mathrm{NDR}$ renormalized mixing matrix elements in $\mathrm{GeV}^{4}$. The first error include all uncertainties other than the uncertaintly due to omitting charm sea quarks, which we estimated to be $2 \%$ and show as the second error.

Light quark discretization effects are tested by: $\mathrm{H})$ adding generic $O\left(\alpha_{s} a^{2}\right)$ terms in addition to the $O\left(\alpha_{s} a^{2}\right)$ taste violating effects in the chiral logarithms. The base fit includes HQ discretization terms of $O\left(\alpha_{s} a\right), O\left(a^{2}\right)$, and $O\left(a^{3}\right)$. Variations include: I) dropping $O\left(a^{3}\right)$ HQ terms, and J) $O\left(a^{2}\right)$ terms as well. The consistency of the continuum extrapolation is test by omitting results, in turn, at either: $\mathrm{K}$ ) the coarsest, or L) finest lattice spacing. The influence of the prior constraints at different orders are tested by: M-O) doubling, in turn, the prior widths. Finally, P) separate fits to each matrix element are done as an alternative to the fit to all five matrix elements.

In Table 2 we list final results for $B$ and preliminary results for $D$ mixing operators. Results are shown for both the BBGLN [34] and BMU [35] matching conventions. The first error is the combined correlated statistical plus systematic error. The second error, due to the absence of charm sea quarks in the gauge ensembles, we estimate to be two percent for the matrix elements.

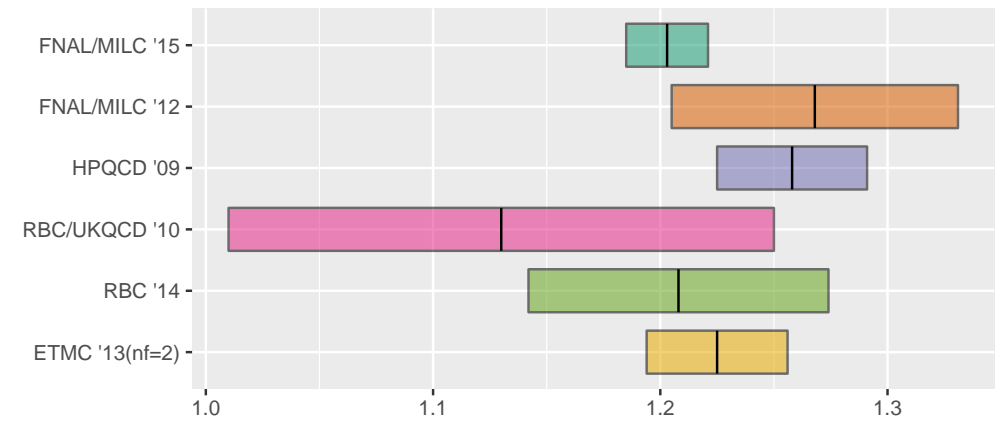

Figure 3: Comparison of the ratio $\xi$ from this work to our previous work [36], and other lattice results. The HPQCD result with asqtad quarks neglects the "wrong spin" contributions to the matrix elements [37]. The $\mathrm{RBC}$ results use a static $b$ quark [38, 39]. The ETMC result is from two-flavor QCD [40].

Both statistical and systematic uncertainties from the lattice calculation tend to cancel in the ratio of the matrix elements. Hence, for $B$ mesons, we compute the isospin breaking ratio,

$$
\xi=\frac{M_{B^{0}}}{M_{B_{s}^{0}}} \sqrt{\frac{\left\langle\mathscr{O}_{1}^{s}\right\rangle}{\left\langle\mathscr{O}_{1}^{d}\right\rangle}}=1.203(17)(6)_{\text {sea }_{c}},
$$

where the second $(0.5 \%)$ error is due the lack of charm sea quarks. Since we account for correlations when taking the ratio of matrix elements the errors are reduced significantly compared to the 
errors in the individual matrix elements. Our value is consistent with our previous result, but now with a significantly smaller error [36]. In Figure 3 we compare our value to other determinations from lattice QCD.

Using $\xi$ we determine the ratio of CKM matrix elements,

$$
\left|\frac{V_{t d}}{V_{t s}}\right|=\xi \sqrt{\frac{\Delta M_{B^{0}} / M_{B^{0}}}{\Delta M_{B_{s}^{0}} / M_{B_{s}^{0}}}} \quad, \quad \text { where } \quad \begin{aligned}
& \Delta M_{B^{0}}=0.5055(20) \mathrm{ps}^{-1} \\
& \Delta M_{B_{s}^{0}}=17.757(21) \mathrm{ps}^{-1}
\end{aligned}
$$

are the experimental world-averages for the mixing frequencies [41]. We find

$$
\left|\frac{V_{t d}}{V_{t s}}\right|=0.2047(4)_{\exp }(29)_{\mathrm{th}_{\mathrm{th}}}(10)_{\mathrm{sea}_{c}}
$$

This result and recent lattice results for $\left|V_{c b}\right|$ and $\left|V_{u b}\right|$ from semileptonic decays tighten the previous constraints on CKM matrix unitarity [42].

\section{Outlook: bag parameters and decay constants}
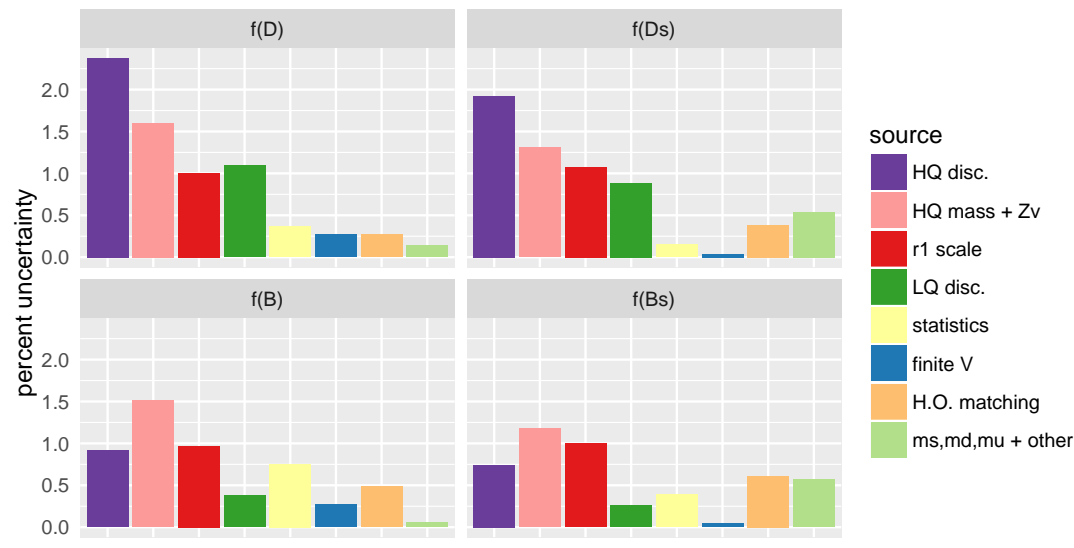

Figure 4: Preliminay analysis of sources of uncertainty for the decay constants. Reference [43] provides details of the decay constant analysis.

We aim to compute bag parameters for the mixing matrix elements using decay constants computed on the same gauge ensembles. We do not present values for the bag parameters since the values of the decay constants remain blinded pending finalization of the analysis of systematic effects. In Figure 4 we present preliminary estimates for the decay constant uncertainties. The coordinated analysis of the decay constants with the mixing matrix elements is in progress.

\section{Acknowledgments}

Computations for this work were carried out with resources provided by the USQCD Collaboration; by the ALCF and NERSC, which are funded by the U.S. Department of Energy (DOE); and by NCAR, NCSA, NICS, TACC, and Blue Waters, which are funded through the U.S. National Science Foundation (NSF). Authors of this work were also supported in part through individual grants by the DOE and NSF (U.S); by MICINN, the Ramón y Cajal program, and the Junta de Andalucía (Spain); by the European Commission; and by the German Excellence Initiative. 


\section{References}

[1] M. Beneke et al., Phys.Lett. B459, 631-640 (1999), [arXiv:hep-ph / 9808385 ].

[2] A. J. Buras et al., Nucl.Phys. B586, 397-426 (2000), [arXiv:hep-ph / 0005183 ].

[3] C. M. Bouchard et al., PoS LATTICE2014, 378 (2014), [arXiv:1412. 5097].

[4] C. C. Chang et al., PoS LATTICE2014, 384 (2014), [arXiv:1411. 6086].

[5] C. Aubin et al., 2015, $10.15484 / \mathrm{milc}$.asqtad.en24a/1177873.

[6] C. Aubin et al., 2015, 10.15484/milc.asqtad.en18a/1178033.

[7] C. Aubin et al., 2015, 10.15484/milc.asqtad.en18b/1178034.

[8] C. Aubin et al., 2015, 10.15484/milc.asqtad.en19a/1178035.

[9] C. Aubin et al., 2015, 10.15484/milc.asqtad.en20a/1178036.

[10] C. Aubin et al., 2015, 10.15484/milc. asqtad.en20b/1178037.

[11] C. Aubin et al., 2015, $10.15484 / \mathrm{milc}$.asqtad.en23a/1178040.

[12] C. Aubin et al., 2015, $10.15484 / \mathrm{milc}$. asqtad.en23b/1178041.

[13] C. Aubin et al., 2015, $10.15484 / \mathrm{milc}$.asqtad.en12a/1178091.

[14] C. Aubin et al., 2015, 10.15484/milc.asqtad.en13a/1178092.

[15] C. Aubin et al., 2015, $10.15484 / \mathrm{milc}$. asqtad.en13b/1178093.

[16] C. Aubin et al., 2015, $10.15484 / \mathrm{milc}$.asqtad.en14a/1178094.

[17] C. Aubin et al., 2015, 10.15484/milc.asqtad.en15a/1178095.

[18] C. Aubin et al., 2015, 10.15484/milc.asqtad.en15b/1178096.

[19] C. Aubin et al., 2015, $10.15484 / \mathrm{milc}$. asqtad.en15c/1178097.

[20] C. Aubin et al., 2015, 10.15484/milc.asqtad.en17a/1178099.

[21] C. Aubin et al., 2015, 10.15484/milc.asqtad.en04a/1178155.

[22] C. Aubin et al., 2015, $10.15484 / \mathrm{milc}$. asqtad.en05a/1178156.

[23] C. Aubin et al., 2015, 10.15484/milc. asqtad.en05b/1178157.

[24] C. Aubin et al., 2015, 10.15484/milc.asqtad.en06a/1178158.

[25] C. Aubin et al., 2015, 10.15484/milc.asqtad.en06b/1178159.

[26] C. Aubin et al., 2015, 10.15484/milc.asqtad.en09a/1178163.

[27] C. Aubin et al., 2015, 10.15484/milc.asqtad.en09b/1178164.

[28] A. Bazavov et al., Rev. Mod. Phys. 82, 1349-1417 (2010), [arXiv:0 903.3598 ].

[29] C. C. Chang et al., (2013), [arXiv:1311.6820].

[30] A. X. El-Khadra et al., Phys.Rev. D64, 014502 (2001), [arXiv:hep-ph/ 0101023 ].

[31] C. Bernard, Phys.Rev. D87, 114503 (2013), [arXiv:1303.0435].

[32] C. Bouchard et al., Phys.Rev. D90, 054506 (2014), [arXiv:14 06.2279 ].

[33] G. P. Lepage et al., Nucl. Phys. Proc. Suppl. 106, 12-20 (2002), [arXiv:hep-lat / 0110175$].$

[34] M. Beneke et al., Nucl.Phys. B639, 389-407 (2002), [arXiv:hep-ph/ 0202106 ].

[35] A. Lenz et al., JHEP 0706, 072 (2007), [arXiv:hep-ph / 0 612167].

[36] A. Bazavov et al., Phys. Rev. D86, 034503 (2012), [arXiv:1205. 7013].

[37] E. Gamiz et al., Phys. Rev. D80, 014503 (2009), [arXiv:0 902.1815$].$

[38] C. Albertus et al., Phys. Rev. D82, 014505 (2010), [arXiv: 1001.2023 ].

[39] Y. Aoki et al., Phys. Rev. D91, 114505 (2015), [arXiv:1406.6192].

[40] N. Carrasco et al., JHEP 03, 016 (2014), [arXiv:1308.1851].

[41] Y. Amhis et al., (2014), [arXiv:1412.7515].

[42] C. DeTar, (2015), [arXiv:1511.06884].

[43] E. T. Neil et al., PoS LATTICE2014, 383 (2015), [arXiv:1501.01991]. 